\title{
STIM1 and Orai1: novel targets for vascular diseases?
}

\author{
Wei ZHANG \& Mohamed TREBAK* \\ Center for Cardiovascular Sciences, Albany Medical College, Albany NY 12208, USA
}

Received May 9, 2011; accepted June 12, 2011

\begin{abstract}
The past five years have witnessed the discovery of the endoplasmic reticulum calcium $\left(\mathrm{Ca}^{2+}\right)$ sensor STIM1 and the plasma membrane $\mathrm{Ca}^{2+}$ channel Orail as the bona fide molecular components of the store-operated $\mathrm{Ca}^{2+}$ entry (SOCE) and the $\mathrm{Ca}^{2+}$ release-activated $\mathrm{Ca}^{2+}$ current $\left(I_{\mathrm{CRAC}}\right)$. It has been known for two decades that SOCE and $I_{\mathrm{CRAC}}$ are required for lymphocyte activation as evidenced by severe immunodeficient phenotypes in patients lacking $I_{\text {CRAC }}$. In recent years however, studies have uncovered expression of STIM1 and Orai1 proteins in various tissues and described additional roles for these proteins in physiological functions and pathophysiological conditions. Here, we will summarize novel findings pertaining to the role of STIM1 and Orai1 in the vascular system and discuss their potential use as targets in the therapy of vascular disease.
\end{abstract}

calcium signaling, calcium channels, Orai1, STIM1, CRAC channels, vascular disease

Citation: Zhang W, Trebak M. STIM1 and Orai1: novel targets for vascular diseases? Sci China Life Sci, 2011, 54: 780-785, doi: 10.1007/s11427-011-4206-6

Calcium $\left(\mathrm{Ca}^{2+}\right)$ ions are universal second messengers and, unlike other second messengers, they are generally located outside the cells or stored in cell organelles such as the endoplasmic reticulum (ER). Resting cytoplasmic $\mathrm{Ca}^{2+}$ concentrations are maintained at $100-300 \mathrm{nmol} \mathrm{L}^{-1}$ [1-3], orders of magnitude lower than the extracellular $\mathrm{Ca}^{2+}$ concentrations (1-2 mmol L ${ }^{-1}$ ). The ER lumen for instance usually maintains $\mathrm{Ca}^{2+}$ concentrations of around $500 \mu \mathrm{mol} \mathrm{L}{ }^{-1}$ and is responsible for transient cytoplasmic $\mathrm{Ca}^{2+}$ increases upon extracellular stimulations. However, a sustained $\mathrm{Ca}^{2+}$ elevation requires $\mathrm{Ca}^{2+}$ entry from the extracellular space through $\mathrm{Ca}^{2+}$ channels located in the plasma membrane (PM). The $\mathrm{Ca}^{2+}$ entry happens concomitantly to the $\mathrm{Ca}^{2+}$ release from $\mathrm{ER}$ lumen and, in some cases, $\mathrm{Ca}^{2+}$ entry is triggered by $\mathrm{Ca}^{2+}$ release. The $\mathrm{Ca}^{2+}$ entry pathway that is specifically activated as a consequence of store emptying is called store-operated $\mathrm{Ca}^{2+}$ entry (SOCE) or capacitative $\mathrm{Ca}^{2+}$ entry [4-8], and the best characterized store-operated $\mathrm{Ca}^{2+}$ current is the calcium release-activated calcium current ( $I_{\text {CRAC }}$ ) [9]. Recent studies have identified the major protein compo-

*Corresponding author (email: trebakm@mail.amc.edu) nents of the SOCE pathway: the $\mathrm{Ca}^{2+}$ sensor protein STIM1 $[10,11]$ and the $\mathrm{Ca}^{2+}$ channel protein Orai1 $[12,13]$.

Calcium release from ER lumen is achieved either actively through physiological agonist-mediated $\mathrm{IP}_{3}$ production and subsequent activation of $\mathrm{IP}_{3}$ receptors or passively by pharmacological inhibition of the sarco-endoplasmic reticulum $\mathrm{Ca}^{2+}$-ATPase (SERCA) on the ER membrane by drugs such as thapsigargin. Store depletion causes the $\mathrm{Ca}^{2+}$ sensor STIM1 on the ER membrane to oligomerize and move to regions of close ER-PM junctions. Orail channels on the plasma membrane move to the same ER-PM junctions and are activated by STIM1 oligomers through direct interaction of a minimal C-terminal 100 amino acid region of STIM1 to the C- and N-termini of Orai1 [14]. Ectopic expression of the minimal 100 amino acid region of STIM1 which was termed SOAR (for STIM Orai Activating Region) or CAD (for CRAC activation Domain) $[15,16]$ is sufficient to activate Orail channels and induce $\mathrm{Ca}^{2+}$ entry independently of store depletion.

There are other members of the STIM and Orai families encoded by separate genes: STIM2, Orai2 and Orai3. STIM2 appears to be involved in maintaining resting $\mathrm{Ca}^{2+}$ 
concentrations. Orai2 and Orai3 are activated by STIM proteins in a similar manner to Orail when co-expressed in HEK293 cells $[14,17,18]$. Orai3 has been shown to encode a native $I_{\mathrm{CRAC}}$-like conductance in breast cancer cells [19], and to contribute to heteromultimeric Orai3/Orai1 storeindependent arachidonate-regulated $\mathrm{Ca}^{2+}$ channels [20]. The role of Orai2 in encoding native $\mathrm{Ca}^{2+}$ entry pathways remains unclear. $\mathrm{Ca}^{2+}$ signaling and SOCE play an essential role in diverse cell functions and are involved in cellular dysfunctions, including those of the vascular system.

\section{Vascular smooth muscle cells and vascular proliferative diseases}

Vascular smooth muscle cells (VSMCs) are the most prominent cell type in blood vessel wall and are responsible for the maintenance of vascular tone [21,22]. VSMCs disorders, usually manifested as a phenotype change, are involved in the pathogenesis of major vascular diseases such as atherosclerosis, hypertension and restenosis. Mechanical or inflammatory stimuli to the blood vessel site cause VSMCs to dedifferentiate from the fully mature quiescent contractile phenotype to an immature synthetic, proliferative and migratory phenotype. Synthetic VSMCs are mainly responsible for the narrowing of blood vessels in atherosclerosis $[23,24]$ and restenosis [25] due to the proliferative/migratory VSMCs accumulation within the innermost layer of blood vessel wall. VSMC proliferation also occurs during vessel remodeling characteristic of development and progression of hypertension as increased presence of immature VSMC was found in the arterioles of hypertensive patients [26].

$\mathrm{Ca}^{2+}$ signaling modulates VSMC function, including phenotypic change, proliferation and migration. SOCE was found increased in synthetic VSMCs compared to the quiescent freshly isolated cells and this correlated with increased protein expression of STIM1 and Orai1 [27,28]. Our lab has demonstrated STIM1 and Orail are essential components of SOCE and $I_{\mathrm{CRAC}}$ in rat VSMCs [28]. The knockdown of STIM1 or Orail greatly reduced thapsigarginor platelet derived growth factor (PDGF)-activated SOCE, as well as VSMC proliferation and migration [28,29]. STIM2, Orai2, Orai3, TRPC1, TRPC4 or TRPC6 knockdown did not show any effect on SOCE. Studies from other groups have also demonstrated that STIM1 is the major component of SOCE and plays an essential role in the proliferation of various types of VSMCs [30,31]. Using an in vivo model of VSMC proliferation and migration, STIM1 was found up-regulated in injured rat carotid arteries subjected to balloon injury $[29,32,33]$. We reported that the protein expression of Orai1 is upregulated in media and neointima of carotid arteries from balloon-injured rats [29]. When an adenoviral vector encoding a short hairpin RNA (shRNA) against STIM1 was applied at the balloon-injured site to pre- vent STIM1upregulation, neointima formation and the activation of transcription factor NFAT were prevented [32,33]. A recent report from our group demonstrated that prevention of Orail upregulation and $I_{\mathrm{CRAC}}$ activation in the balloon-injured carotid artery of rats using a lentiviral vector encoding shRNA inhibits NFAT nuclear translocation and activity, VSMC proliferation and neointima formation [34].

SOCE was also found increased in metabolic syndrome (MetS) swine coronary smooth muscle cells. MetS is a combination of medical disorders and MetS patients are at higher risk for coronary artery disease, stroke and type 2 diabetes. The swine MetS model consists of 40 weeks on atherogenic diets and stent placement in coronary artery of male Ossabaw pigs [35,36]. The increase of SOCE in MetS smooth muscle cells was associated with increased STIM1, Orai1 and TRPC1 mRNA and protein levels [35]. However, the increase of SOCE and STIM1/TRPC1 protein overexpression were attenuated by subjecting the pigs to exercise training [35].

Giachini et al. [37] have discovered a role for STIM1 and Orail in hypertensive rat aorta. Twenty-four weeks old stroke-prone spontaneously hypertensive rats (SHR) displayed higher systolic blood pressure, compared to WistarKyoto (WKY) rats. Endothelium-denuded aortic rings from SHR displayed greater contractions than WKY rats during the $\mathrm{Ca}^{2+}$ store re-loading period following store depletion. Caffeine-induced SHR aortic contraction was greater than WKY rats', and both contractions were dramatically suppressed by CRAC channel blockers 2-aminophenyl borane (2-APB) and gadolinium $\left(\mathrm{Gd}^{3+}\right)$. Application of Orai1 or STIM1 antibodies reduced the SR $\mathrm{Ca}^{2+}$ loading capacity and the transient aortic contractions of both SHR and WKY rats induced by caffeine and abolished the differences in contractions between SHR and WKY rats. These authors showed higher protein and mRNA levels of STIM1 and Orai1 in SHR aortas. Immuno-fluorescence analysis confirmed the increased expression of STIM1 and Orai1 proteins in SHR aortas [37]. Collectively, the data presented above supports a role for the SOCE pathway and STIM1/ Orail proteins in driving smooth muscle proliferation and migration and suggests the potential use of these proteins as targets for vascular occlusive diseases.

\section{Platelets and thrombosis}

Platelets are small anucleated cells derived from bone marrow megakaryocytes. They are circulating within the blood, being prepared to respond to any damage to the blood vessels. The stimuli released from the injured site of blood vessel walls trigger platelets activation and aggregation, which cause blood coagulation to prevent bleeding (hemostasis). However, when the platelets aggregation and blood coagulation are enhanced in response to severe damage and overwhelming stimuli, the blood clot formed inside the 
vessel lumen obstructs the blood flow rather than heals the injury, and this process is a pathological one called thrombosis. Platelets activation is the major cause of thrombosis and is therefore the major target of antithrombotic therapy. Platelets activation and aggregation requires intracellular $\mathrm{Ca}^{2+}$ influx during the processes of their adhesion to the exposed subendothelium, aggregation and formation of clots in blood vessel lumen. Diverse agonists are involved in platelets activation through elevations of $\mathrm{Ca}^{2+}$ concentrations, mainly through two different pathways [30]. Soluble agonists (thrombin, ADP and thromboxane $\mathrm{A}_{2}$; $\mathrm{TXA}_{2}$ ) activate platelets through $\mathrm{G}$ protein-coupled receptors (P2Y, PAR, and TP respectively). G protein then leads to the activation of phospholipase $\mathrm{C} \beta$ (PLC $\beta$ ) and $\mathrm{IP}_{3}$ production $[38,39]$. Adhesive ligands (von Willebrand factor; vWF, collagen, fibronection, etc.) activate platelet surface receptors such as GPIb/V/IX, GPVI, and integrins, and trigger the production of $\mathrm{IP}_{3}$ by activation of phospholipase $\mathrm{C} \gamma$ (PLC $\gamma$ ) [40]. Although both pathways can presumably activate SOCE by the production of $\mathrm{IP}_{3}$, the $\mathrm{G}$ protein pathway mainly triggers platelet-platelet interaction, while GPIb/ V/XI and GPVI pathways mediate platelets aggregation through platelet-matrix adhesion. The cellular mechanisms of these processes remain largely unknown.

Both STIM1 and Orai1 are found highly expressed in platelets, suggesting they might play an important role in platelet function. Bernhard Nieswandt group has generated three types of SOCE-impaired mice line: STIM1 ${ }^{\text {sax/sax }}$ (an activating STIM1 EF hand D84G mutant), $\mathrm{STIM}^{-1-}$, and Orai $^{-l-}[41-43]$. The STIM1 ${ }^{\text {sax/sax }}$ homozygous mice embryos had severe hemorrhage and only a few of them survived until E13-E14. Due to the high lethality and low survival of homozygous animals (1 out of 72 offspring), most of the work was done in the STIM $1^{\text {sax/+ }}$ heterozygous mice in which the impairment of SOCE and platelets function was also observed. Platelets from STIM $1^{\mathrm{sax} /+}$ mice were pre-activated, displayed elevated basal $\mathrm{Ca}^{2+}$ concentrations and a short life span. $\mathrm{Ca}^{2+}$ store release evoked by thapsgargin (TG) was reduced by $60 \%$ in the platelets from these heterozygous animals and the subsequent $\mathrm{Ca}^{2+}$ influx (in the presence of $2 \mathrm{mmol} \mathrm{L}^{-1}$ extracellular $\mathrm{Ca}^{2+}$ ) was reduced by $70 \%$ compared with wild type. One important mechanism was discovered when the authors compared the $\mathrm{Ca}^{2+}$ influx in response to agonists in STIM1 $1^{\mathrm{sax} /+}$ and wild type platelets. They found that $\mathrm{Ca}^{2+}$ influx in STIM1 mutant platelets was reduced only upon stimulation with collagen receptor-specific agonists (such as collagen related peptide; CRP and rhodocytin; RC) [42]. Those receptors are associated with the immunoreceptor tyrosine-based activating motif (ITAM) and trigger $\mathrm{Ca}^{2+}$ influx through activation of the PLC $\gamma$ pathway, reminiscent of $T$ cell receptor activation [44]. In contrast, when $\mathrm{G}$ protein coupled agonists such as thrombin and ADP were applied, levels of $\mathrm{Ca}^{2+}$ rise were similar in both mutant and wild type platelets [42]. The STIM $1^{\text {sax/+ }}$ mice blood also showed less adhesion to the collagen surface than wild type.
Tail bleeding times were significantly prolonged in $\mathrm{STIM}^{\mathrm{sax} /+}$ mice. One specific injury model was used, in which the thrombus formation is mainly driven by thrombin, and the formation times of occlusion were similar in STIM $1^{\text {sax/+ }}$ mice and wild type mice [42].

In the $\mathrm{STIM}^{-/-}$mice platelets, $\mathrm{Ca}^{2+}$ influx evoked by either CRP or by the $\mathrm{G}$ protein-coupled agonists (ADP, thrombin and $\mathrm{TXA}_{2}$ analogue U46619) was suppressed. However, in a manner similar to STIM $1^{\mathrm{sax} /+}$ mice, platelets aggregation was only diminished in $\mathrm{STIM}^{-/-}$blood when triggered by collagen-related agonists, and did not change in $\mathrm{STIM}^{-1-}$ blood when triggered by $\mathrm{G}$ protein-coupled agonists, compared to wild type. The experiments of three-dimensional growth of thrombi on collagen-coated surface showed that STIM1 ${ }^{-1-}$ platelets formed less thrombus than the wild type did. Surface area covered by mutant platelets was reduced by $\sim 42 \%$, and the total volume of thrombus formed by mutant platelets was reduced by $\sim 81 \%$. In vivo experiments showed a mild prolongation of tail bleeding time, a significant delay in vessel occlusion time and a high resistance to ischemic brain infarction in $\mathrm{STIM}^{-1-}$ mice [43].

Orail was found to be the predominant member of the Orai family in both human and mice platelets. Because the Orai1 knockout mice showed very high mortality, Braun et al. [41] transplanted Orai1 ${ }^{-/-}$bone marrow to irradiated wild-type mice and generated platelets Orai $1^{-1-}$ chimeric mice. Thapsigargin-evoked SOCE was dramatically suppressed in platelets, indicating that Orail is the essential component of SOCE in these cells. Very similar to $\mathrm{STIM}^{-1-}$ platelets, the $\mathrm{Ca}^{2+}$ influx of Orai1 ${ }^{-/-}$platelets was inhibited in response to CRP, ADP and thrombin. However, the Orai ${ }^{-1-}$ platelets aggregation in response to $G$ protein-coupled agonists (ADP and thrombin) was comparable to wild type, but was diminished in response to low concentration of collagen or CRP. Intravenous injection of collagen/epinephrine caused death of wild type mice within 20 min by pulmonary thromboembolism, whereas most Orai $^{-1-}$ mice (6 out of 7) survived [41]. In an arterial thrombosis model, whereas all the wild type mice got complete occlusion, 4 out of 10 Orai1knockout mice had maintained blood flow. In a $\mathrm{FeCl}_{3}$-induced arterioles injury model, where the thrombus formation mainly depends on thrombin, 14 out of 15 Orai $^{-1-}$ mice had occlusive thrombi and the process was similar to wild type mice. Similar to the STIM $1^{-1-}$ mice, the Orai $1^{-1-}$ mice showed high resistance to ischemic brain infarction [41]. Around the same time, Stefan Feske's group generated chimeric mice expressing Orai1 ${ }^{\mathrm{R} 93 \mathrm{~W}}$ exclusively in blood cells [45]; Orai1 ${ }^{\mathrm{R} 93 \mathrm{~W}}$ is the mutation of Orai1 found in severe combined immunodeficient patients (SCID) characterized by abrogated SOCE and $I_{\mathrm{CRAC}}[46] . \mathrm{Ca}^{2+}$ entry was impaired when Orai $1^{\mathrm{R} 93 \mathrm{~W}}$ platelets were stimulated by either thapsigargin or agonists (PAR4p or convulxin to active PAR4 or GPVI respectively). However, platelet aggregation 
and thrombus formation was not apparently affected [45]. Since mice harboring Orai ${ }^{\mathrm{R} 93 \mathrm{~W}}$ mutation are equivalent to human SCID patients with Orai1 ${ }^{\mathrm{R} 91 \mathrm{~W}}$ mutation and these patients did not show any obvious bleeding or clotting issues, these results are consistent with the clinical observations. However, these authors found that exposure of phosphatidylserine (PS) on cell surface of Orai1 ${ }^{\mathrm{R} 91 \mathrm{~W}}$ platelets was reduced by $80 \%$ [45]. The cell surface exposure of PS is required for platelet procoagulant activity and is triggered by cytosolic $\mathrm{Ca}^{2+}$ elevation. These results were confirmed by the Nieswandt group where PS exposure and thrombus generation was suppressed in $\mathrm{STIM}^{-/-}$and Orai $1^{-/-}$mice when triggered through the GPVI pathway (using convulxin as an agonist) but not through thrombin stimulation; STIM2 did not show any apparent role in these processes [47].

To summarize, STIM1 and Orai1 are the major components of SOCE in platelets and dominate the $\mathrm{Ca}^{2+}$ Entry through GPIb-GPVI-ITAM signaling pathway. Deficiency of STIM1 or Orai1 reduced thrombosis formation in arteries (subjected to high shear flow conditions) when triggered mainly by collagen (through GPVI/ PLC $\gamma$ pathway). In contrast, STIM1 and Orail did not show any evident effect in hemostasis at the wound bleeding site, where abundant tissue factors are involved and thrombus formation is triggered mainly by thrombin or other tissue factors acting through $G$ protein coupled receptors involving the activation of the PLC $\beta$ pathway.

\section{Endothelial cells and angiogenesis}

Endothelial cells line the inner side of blood vessels and help maintain vascular tone through production of nitric oxide and act as a barrier between the circulating blood and the surrounding tissue. Endothelial dysfunction is involved in various cardiovascular diseases.

Endothelial cells have been known for a while to express the SOCE pathway $[48,49]$ and the elevation of $\mathrm{Ca}^{2+}$ concentration in cytoplasm plays an important role in a variety of endothelial cell functions, such as barrier function, motility, proliferation, migration and angiogenesis [50-54]. Work in our lab showed that endothelial cells from different vascular beds, human umbilical vein endothelial cells (HUVEC) and human pulmonary artery endothelial cells (HPAEC) express STIM and Orai proteins and display SOCE and $I_{\text {CRAC }}$ that are encoded by STIM1 and Orai1 [55]. Interestingly, endothelial agonists such as vascular endothelial growth factor (VEGF) or thrombin regulate intracellular calcium entry through STIM1/Orai1-mediated SOCE [55]. STIM1/Orai1-mediated SOCE plays an essential role in HUVEC proliferation; STIM1 or Orai1 knockdown using silencing RNA (siRNA) leads to cell cycle arrest at S and G2/M phases of the cell cycle [55]. Orail and SOCE are also functional in HUVEC angiogenesis [56]. Knockdown of Orai1 or STIM1 by siRNA suppressed VEGF-evoked
$\mathrm{Ca}^{2+}$ entry and inhibited HUVEC migration through $8-\mu \mathrm{m}$ pores. Orail was pivotal for tube formation of HUVEC in Matrigel, suggesting Orail as a potential target in angiogenesis. A CRAC channel inhibitor, S66, showed similar effect on tube formation and also inhibited vessel growth in an in vivo model of angiogenesis [56]. SOCE was also found expressed in human Endothelial Progenitor Cells (EPC) [57], including those harvested from peripheral blood (PB-EPC) and umbilical cord blood (UCB-EPC). Orai1 and STIM1 were more abundant in both types of EPCs, and were proposed as the major components of SOCE. Knockdown of STIM1 attenuated hepatocyte growth factor (HGF)-induced SOCE and proliferation in EPC [58].

\section{Cardiac function and hypertrophy}

Ohba et al. [59] demonstrated that rat cardiomyocytes expressed STIM1 and that knockdown of STIM1 inhibited $\mathrm{Ca}^{2+}$ entry in response to thapsigargin and endothelin-1. Importantly, STIM1 knockdown inhibited endothelin1-mediated nuclear factor for activated T-cells (NFAT) activation and prevented the increase of cardiac fetal genes induced by hypertrophic stimuli such as endothelin-1, phenyl-epinephrine [59]. Voelkers et al. [60] demonstrated that STIM1 and Orai1 proteins are required for thapsigargin-mediated SOCE and $\mathrm{Ca}^{2+}$ transients in neonatal cardiomyocytes. These authors also showed that knockdown of Orail caused a significant decrease in cardiac fetal genes expression and size of neonatal cardiomyocytes and a reduction in ERK1/2 phosphorylation and calcineurin activation under resting conditions and hypertrophic stimulation with phenylepinephrine [60]. However, STIM1 knockdown caused a significant decrease in cell size only in response to hypertrophic stimulation with phenylephrine, but had no effect under resting conditions. These data support a role for STIM1 and Orai1 in hypertrophic cardiac remodeling [60].

\section{Conclusion}

STIM1 and Orai1 have recently emerged as mediators for SOCE in several cell types including those of the cardiovascular system. The contribution of STIM1 and Orai1 to cardiovascular diseases is also beginning to emerge. As of this writing, there is no indication for a role of Orai2 or Orai3 in the cardiovascular system but future research is likely to unravel novel roles for these isoforms. Orai isoforms are known to heteromultimerize, suggesting means to enhance the diversity of calcium signals induced by specific agonists to control specific physiological functions. We look forward to future work to unravel additional roles, oligomerization patterns and regulation mechanisms for native STIM and Orai proteins in different cells of the cardiovascular system. Deciphering subtle differences of Orai 
channel organization and regulation between different cell types might make selective targeting of these molecules in treatment of cardiovascular diseases a reality.

This work was supported by the National Institutes of Health (Grant No. 5R01HL097111) to Mohamed Trebak.

1 Hogan P G, Lewis R S, Rao A. Molecular basis of calcium signaling in lymphocytes: STIM and ORAI. Annu Rev Immunol, 2010, 28: 491-533

2 House S J, Potier M, Bisaillon J, et al. The non-excitable smooth muscle: calcium signaling and phenotypic switching during vascular disease. Pflugers Arch, 2008, 456: 769-785

3 Wamhoff B R, Bowles D K, Owens G K. Excitation-transcription coupling in arterial smooth muscle. Circ Res, 2006, 98: 868-878

4 Frischauf I, Schindl R, Derler I, et al. The STIM/Orai coupling machinery. Channels (Austin), 2008, 2: 261-268

5 Potier M, Trebak M. New developments in the signaling mechanisms of the store-operated calcium entry pathway. Pflugers Arch, 2008, 457: 405-415

6 Putney J W. Capacitative calcium entry: from concept to molecules. Immunol Rev, 2009, 231: 10-22

7 Putney J W Jr. Capacitative calcium entry revisited. Cell Calcium, 1990, 11: 611-624

8 Putney J W Jr. A model for receptor-regulated calcium entry. Cell Calcium, 1986, 7: 1-12

9 Hoth M, Penner R. Depletion of intracellular calcium stores activates a calcium current in mast cells. Nature, 1992, 355: 353-356

10 Liou J, Kim M L, Heo W D, et al. STIM is a $\mathrm{Ca}^{2+}$ sensor essential for $\mathrm{Ca}^{2+}$-store-depletion-triggered $\mathrm{Ca}^{2+}$ influx. Curr Biol, 2005, 15: $1235-1241$

11 Roos J, DiGregorio P J, Yeromin A V, et al. STIM1, an essential and conserved component of store-operated $\mathrm{Ca}^{2+}$ channel function. J Cell Biol, 2005, 169: 435-445

12 Feske S, Gwack Y, Prakriya M, et al. A mutation in Orail causes immune deficiency by abrogating CRAC channel function. Nature, 2006, 441: 179-185

13 Vig M, Peinelt C, Beck A, et al. CRACM1 is a plasma membrane protein essential for store-operated $\mathrm{Ca}^{2+}$ entry. Science, 2006, 312: $1220-1223$

14 DeHaven W I, Smyth J T, Boyles R R, et al. Calcium inhibition and calcium potentiation of Orai1, Orai2, and Orai3 calcium releaseactivated calcium channels. J Biol Chem, 2007, 282: 17548-17556

15 Park C Y, Hoover P J, Mullins F M, et al. STIM1 clusters and activates CRAC channels via direct binding of a cytosolic domain to Orai1. Cell, 2009, 136: 876-890

16 Yuan J P, Zeng W, Dorwart M R, et al. SOAR and the polybasic STIM1 domains gate and regulate Orai channels. Nat Cell Biol, 2009, 11: $337-343$

17 Frischauf I, Muik M, Derler I, et al. Molecular determinants of the coupling between STIM1 and Orai channels: differential activation of Orai1-3 channels by a STIM1 coiled-coil mutant. J Biol Chem, 2009, 284: $21696-21706$

18 Schindl R, Frischauf I, Bergsmann J, et al. Plasticity in $\mathrm{Ca}^{2+}$ selectivity of Orai1/Orai3 heteromeric channel. Proc Natl Acad Sci USA, 2009, 106: 19623-19628

19 Motiani R K, Abdullaev I F, Trebak M. A novel native store-operated calcium channel encoded by Orai3: selective requirement of Orai3 versus Orai1 in estrogen receptor-positive versus estrogen receptornegative breast cancer cells. J Biol Chem, 2010, 285: 19173-19183

20 Mignen O, Thompson J L, Shuttleworth T J. Both Orai1 and Orai3 are essential components of the arachidonate-regulated $\mathrm{Ca}^{2+}$-selective (ARC) channels. J Physiol, 2008, 586: 185-195

21 Wray S, Burdyga T. Sarcoplasmic reticulum function in smooth muscle. Physiol Rev, 2010, 90: 113-178

22 Wray S, Burdyga T, Noble K. Calcium signalling in smooth muscle.
Cell Calcium, 2005, 38: 397-407

23 Orr A W, Hastings N E, Blackman B R, et al. Complex regulation and function of the inflammatory smooth muscle cell phenotype in atherosclerosis. J Vasc Res, 2010, 47: 168-180

24 Ross R, Glomset J A. Atherosclerosis and the arterial smooth muscle cell: Proliferation of smooth muscle is a key event in the genesis of the lesions of atherosclerosis. Science, 1973, 180: 1332-1339

25 Liu M W, Roubin G S, King S B 3rd. Restenosis after coronary angioplasty. Potential biologic determinants and role of intimal hyperplasia. Circulation, 1989, 79: 1374-1387

26 Puato M, Faggin E, Favaretto E, et al. Prevalence of fetal-type smooth muscle cells in the media of microvessels from hypertensive patients. Hypertension, 2004, 44: 191-194

27 Berra-Romani R, Mazzocco-Spezzia A, Pulina M V, et al. $\mathrm{Ca}^{2+}$ handling is altered when arterial myocytes progress from a contractile to a proliferative phenotype in culture. Am J Physiol Cell Physiol, 2008, 295: C779-790

28 Potier M, Gonzalez J C, Motiani R K, et al. Evidence for STIM1- and Orail-dependent store-operated calcium influx through ICRAC in vascular smooth muscle cells: role in proliferation and migration. FASEB J, 2009, 23: 2425-2437

29 Bisaillon J M, Motiani R K, Gonzalez-Cobos J C, et al. Essential role for STIM1/Orai1-mediated calcium influx in PDGF-induced smooth muscle migration. Am J Physiol Cell Physiol, 2010, 298: C993-1005

30 Li J, Sukumar P, Milligan C J, et al. Interactions, functions, and independence of plasma membrane STIM1 and TRPC1 in vascular smooth muscle cells. Circ Res, 2008, 103: e97-104

31 Takahashi Y, Watanabe H, Murakami M, et al. Functional role of stromal interaction molecule 1 (STIM1) in vascular smooth muscle cells. Biochem Biophys Res Commun, 2007, 361: 934-940

32 Aubart F C, Sassi Y, Coulombe A, et al. RNA interference targeting STIM1 suppresses vascular smooth muscle cell proliferation and neointima formation in the rat. Mol Ther, 2009, 17: 455-462

33 Guo R W, Wang H, Gao P, et al. An Essential role for STIM1 in neointima formation following arterial injury. Cardiovasc Res, 2009, 81: 660-668

34 Zhang W, Halligan $\mathrm{K}$ E, Zhang $\mathrm{X}$, et al. Orai1-mediated $I_{\mathrm{CRAC}}$ is essential for neointima formation after vascular injury. Circ Res, 2011 (in Press)

35 Edwards J M, Neeb Z P, Alloosh M A, et al. Exercise training decreases store-operated $\mathrm{Ca}^{2+}$ entry associated with metabolic syndrome and coronary atherosclerosis. Cardiovasc Res, 2010, 85: 631-640

36 Neeb Z P, Edwards J M, Alloosh M, et al. Metabolic syndrome and coronary artery disease in Ossabaw compared with Yucatan swine. Comp Med, 2010, 60: 300-315

37 Giachini F R, Chiao C W, Carneiro F S, et al. Increased activation of stromal interaction molecule-1/Orai-1 in aorta from hypertensive rats: a novel insight into vascular dysfunction. Hypertension, 2009, 53: $409-416$

38 Hagedorn I, Vogtle T, Nieswandt B. Arterial thrombus formation. Novel mechanisms and targets. Hamostaseologie, 2010, 30: 127-135

39 Li Z, Delaney M K, O’Brien K A, et al. Signaling during platelet adhesion and activation. Arterioscler Thromb Vasc Biol, 2010, 30: 2341-2349

40 Varga-Szabo D, Braun A, Nieswandt B. Calcium signaling in platelets. J Thromb Haemost, 2009, 7: 1057-1066

41 Braun A, Varga-Szabo D, Kleinschnitz C, et al. Orai1 (CRACM1) is the platelet SOC channel and essential for pathological thrombus formation. Blood, 2009, 113: 2056-2063

42 Grosse J, Braun A, Varga-Szabo D, et al. An EF hand mutation in Stim1 causes premature platelet activation and bleeding in mice. $\mathrm{J}$ Clin Invest, 2007, 117: 3540-3550

43 Varga-Szabo D, Braun A, Kleinschnitz C, et al. The calcium sensor STIM1 is an essential mediator of arterial thrombosis and ischemic brain infarction. J Exp Med, 2008, 205: 1583-1591

44 Oh-Hora M, Yamashita M, Hogan P G, et al. Dual functions for the endoplasmic reticulum calcium sensors STIM1 and STIM2 in T cell activation and tolerance. Nat Immunol, 2008, 9: 432-443

45 Bergmeier W, Oh-Hora M, McCarl C A, et al. R93W mutation in 
Orai 1 causes impaired calcium influx in platelets. Blood, 2009, 113: 675-678

46 Feske S. CRAC channelopathies. Pflugers Arch, 2010, 460: 417-435

47 Gilio K, van Kruchten R, Braun A, et al. Roles of platelet STIM1 and Orai1 in glycoprotein VI- and thrombin-dependent procoagulant activity and thrombus formation. J Biol Chem, 2010, 285: 23629-23638

48 Fasolato C, Nilius B. Store depletion triggers the calcium releaseactivated calcium current (ICRAC) in macrovascular endothelial cells: a comparison with Jurkat and embryonic kidney cell lines. Pflugers Arch, 1998, 436: 69-74

49 Schilling W P, Cabello O A, Rajan L. Depletion of the inositol 1,4,5-trisphosphate-sensitive intracellular $\mathrm{Ca}^{2+}$ store in vascular endothelial cells activates the agonist-sensitive $\mathrm{Ca}(2+)$-influx pathway. Biochem J, 1992, 284: 521-530

50 Antoniotti S, Fiorio Pla A, Pregnolato S, et al. Control of endothelial cell proliferation by calcium influx and arachidonic acid metabolism: a pharmacological approach. J Cell Physiol, 2003, 197: 370-378

51 Munaron L. Intracellular calcium, endothelial cells and angiogenesis. Recent Pat Anticancer Drug Discov, 2006, 1: 105-119

52 Munaron L, Fiorio Pla A. Endothelial calcium machinery and angiogenesis: understanding physiology to interfere with pathology. Curr Med Chem, 2009, 16: 4691-4703

53 Tiruppathi C, Ahmmed G U, Vogel S M, et al. $\mathrm{Ca}^{2+}$ signaling, TRP channels, and endothelial permeability. Microcirculation, 2006, 13:
693-708

54 Tiruppathi C, Minshall R D, Paria B C, et al. Role of $\mathrm{Ca}^{2+}$ signaling in the regulation of endothelial permeability. Vascul Pharmacol, 2002, 39: $173-185$

55 Abdullaev I F, Bisaillon J M, Potier M, et al. Stim1 and Orai1 mediate CRAC currents and store-operated calcium entry important for endothelial cell proliferation. Circ Res, 2008, 103: 1289-1299

56 Li J, Cubbon R M, Wilson L A, et al. Orai1 and CRAC channel dependence of VEGF-activated $\mathrm{Ca}^{2+}$ entry and endothelial tube formation. Circulation Research advance online publication 13 May 2011; doi: 10.1161/CIRCRESAHA.111.243352

57 Sanchez-Hernandez Y, Laforenza U, Bonetti E, et al. Store-operated $\mathrm{Ca}(2+)$ entry is expressed in human endothelial progenitor cells. Stem Cells Dev, 2010, 19: 1967-1981

58 Shi Y, Song M, Guo R, et al. Knockdown of stromal interaction molecule 1 attenuates hepatocyte growth factor-induced endothelial progenitor cell proliferation. Exp Biol Med (Maywood), 2010, 235: 317-325

59 Ohba T, Watanabe H, Murakami M, et al. Essential role of STIM1 in the development of cardiomyocyte hypertrophy. Biochem Biophys Res Commun, 2009, 389: 172-176

60 Voelkers M, Salz M, Herzog N, et al. Orai1 and Stim1 regulate normal and hypertrophic growth in cardiomyocytes. J Mol Cell Cardiol, 2010, 48: 1329-1334

Open Access This article is distributed under the terms of the Creative Commons Attribution License which permits any use, distribution, and reproduction in any medium, provided the original author(s) and source are credited. 